\title{
Dual Effect of Surface Active Substances on the Reduction of Copper (II) Ions
}

\author{
By \\ Taitiro Fujinaga and Satoshi Okazaki \\ (Ghemistry Institute, Faculty of Science, Kyoto University, Kyoto)
}

(Received July 13, 1964)

Surface active substances (SAS) usually employed as maximum suppressors in d.c. polarography often cause an inhibition of the electrode reaction. Since Lingane ${ }^{1)}$ found the abnormal effect of gelatin on the copper wave in sulfuric acid medium, many striking effects of SAS on d.c. polarograms have been reported. Recently, the attempts have been made to explain the origin or nature of these effects by several workers ${ }^{2-9}$. Kolthoff and Okinaka ${ }^{10}$ ) were the first to have described the accelerative effect of anionic SAS on the electrode reaction of simple cupric ions. Frumkin et al. ${ }^{11)}$ described the dual effect of cationic SAS which accelerated or decelerated the reduction of $\mathrm{PtCl}_{4}{ }^{2-}$ ions depending on its concentration. Tanaka et al. ${ }^{12)}$ also reported the dual effect of cationic SAS on the reduction of manganese (III) ions.

Our present work was carried out in order to investigate the electrode reaction of copper (II) ions in the presence of SAS by means of a.c. polarography. The a.c. polarogram of copper (II) ions in perchlroic acid gave an irreversible wave and its peak height was decreased by the addition of non-ionic or cationic SAS, while increased by the addition of anionic SAS (sodium lauryl sulfate; SLS). The rate constant $\mathrm{k}_{\mathrm{s}}$ for the reduction

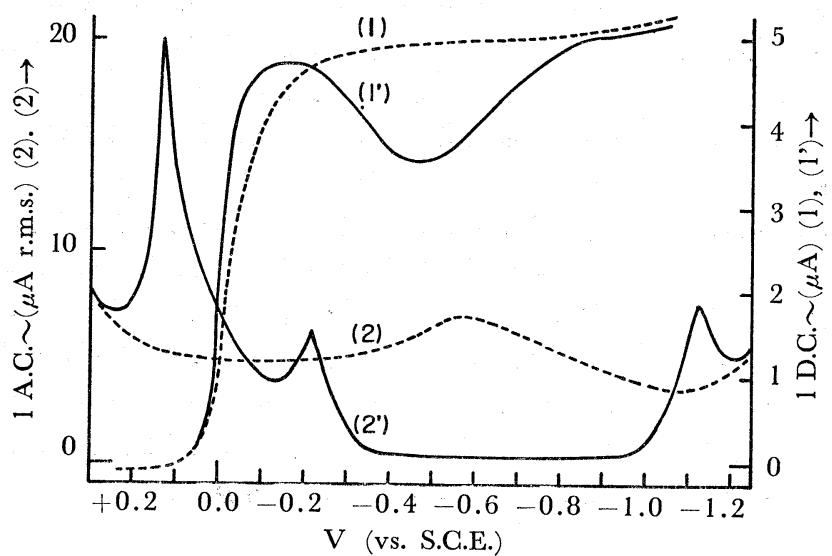

Fig. 1. Effect of SLS on D.C. copper wave and A.C. base current in $1 \mathrm{M}-\mathrm{HClO}_{4}$.

Curves (1), (1') : D.C. copper waves of $1 \times 10^{-3} M-\mathrm{Cu}^{2+}$ in $1 M-\mathrm{HClO}_{4} \cdot(1):$ in the absence of SLS and (1') : in the presence of $0.0075 \%$ SLS.

Curves (2), (2') : A.C. base currents in $1 M-\mathrm{HClO}_{4}$ $(2):$ in the absence of SLS, and $\left(2^{\prime}\right):$ in the presence of $0.0075 \%$ SLS. of $1 \mathrm{~m} M$ copper perchlorate in $1 M$ perchloric acid, which was measured according to the methode of Kambara et al. ${ }^{13)}$, was $1 \times 10^{-3}$ $\mathrm{cm} . \mathrm{sec}^{-}$in the absence of SAS and $1 \times 10^{-2} \mathrm{~cm} . \mathrm{sec}^{-1}$ in the presence of $0.02 \%-0.1 \%$ SLS.

In the course of this study, we found that SLS gives a kind of dual effects on the copper (II) wave. Figure 1 shows the relationship between the d.c. polarograms of copper (II) ions and the tensammetric waves of SLS in $1 M$ perchloric acid. In agreement with the results of Kolthoff and 
Okinaka, the d.c. copper wave became steep and the half-wave potential shifted slightly to more positive potentials by the presence of SLS. Moreover, SLS suppressed the diffusion current in a potential range between $-0.2 \mathrm{~V} \sim-0.8 \mathrm{~V}$ vs. S.C.E., which was not reported by Kolthoff and Okinaka. The tensammetric waves of SLS in curve 2' of Figure 1 gave two adsorption peaks at $+0.13 \mathrm{~V}$ and $-0.22 \mathrm{~V}$ (both potentials being more positive than that of the electrocapillary maximum), differed from the usual cases. (We call the the peak appeared at $+0.13 \mathrm{~V}$ 'the first adsorption wave' and that at $-0.22 \mathrm{~V}$ 'the second adsorption wave'.) From this figure, SLS which is a long-chain anionic SAS seems to be adsorbed at the electrode surface through two steps. At the first step, SLS seems to be adsorbed at the positively charged electrode surface by electrostatic force forming the first adsorption film which accelerates the electrode reaction by reason of the so-called Frumkin's double layer effects ${ }^{14}$. When the electrode potential becomes more negative, the first adsorption film seems to be converted suddenly into the second adsorption film whose structure is so compact that the reduction of copper (II) ion is decelerated and the d.c. copper wave is distorted.

As above mentioned, SLS causes the dual effect independently upon the electrode reaction of copper (II) ions according to the change of structure of the adsorption films. From this example, it seems to be very important to investigate the electrode reaction in the presence of SAS by taking into account the structure of adsorption films.

The detailed results will be published elsewhere.

\section{References}

1) J.J. Lingane, Ind. Eng. Chem. Anal. Ed., 15, 584 (1943).

2) L. Meites and T. Meites, J. Am. Chem. Soc., 73, 177 (1951).

3) M. Dratovsky and M. Ebert, Chem. Listy, 48, 498 (1954).

4) A.N. Frumkin, Z. Elektrochem., 59, 807 (1955).

5) R. Tamamushi and T. Yamanaka, Bull. Chem. Soc. Japan, 28, 673 (1955).

6) P. Delahay and I. Trachtenberg, J. Am. Chem. Soc., 79, 2355 (1957).

7) R.W. Schmid and C.N. Reilley, J. Am. Chem. Soc., 80, 2087 (1958).

8) M. Ishibashi, T. Fujinaga and M. Sato, J. Chem. Soc. Japan, 80, 389 (1959).

9) H.W. Nürnberg and M. von Stackelberg, J. Electroanal. Chem., 4, 1 (1962).

10) I.M. Kolthoff and Y. Okinaka, J. Am. Chem. Soc., 81, 2296 (1959).

11) A.N. Frumkin, O.A. Petrii and N.V. Nikolaeva-Fedorich, Doklady Akad. Nauk. SSSR, 136, 1158 (1961).

12) N. Tanaka and Y. Kikuchi, Bull. Chem. Soc. Japan, 36, 759 (1963).

13) T. Kambara and T. Ishii, Rev. Polarography, 9, 30 (1961).

14) A.N. Frumkin, Trans. Faraday Soc., 55, 156 (1959). 